\title{
Réponse des fibroblastes humains dermiques normaux en culture à de très faibles débits de dose d'irradiation chronique
}

\author{
B. PIANEZZI* F. CROUTE* ${ }^{*}$, F. STEINHAUSLER ${ }^{\star *}$, \\ Y. GAUBIN* ${ }^{\star}$ J.P. SOLEILHAVOUP*
}

RÉSUMÉ Des fibroblastes dermiques humains en culture ont été irradiés par une source de ${ }^{60} \mathrm{Co}$ (débit de dose $6,25 \mathrm{mGy} /$ jour) et exposés pendant 8 jours (dose absorbée totale $50 \mathrm{mGy}$ ). La prolifération, les teneurs en protéines et ADN n'ont pas été modifiées sous irradiation. Le potentiel transmembranaire de 200 cellules était comparable dans les cellules irradiées $(9,4 \pm 4,9 \mathrm{eV})$ et les cellules témoins $(10,2 \pm 2,0 \mathrm{eV})$. Les dosages de l'activité de la glucose6-phosphate deshydrogénase (G6P-DH, enzyme-clé de la voie des pentoses phosphates) de la glycéraldéhyde-3-phosphate deshydrogénase (GAP-DH) et de la pyruvate kinase (enzyme-clé de la glycolyse), ont montré que cette irradiation chronique n'induisait pas de changement de l'activité de la G6PDH. Au contraire, les activités de la GAP-DH et de la pyruvate kinase étaient significativement - mais transitoirement - inhibées (jusqu'à $25 \%$ ) au début de la phase exponentielle de croissance $\left(4^{e}, 5^{e}\right.$ jour). L'activité catalasique (enzyme de destruction de $\mathrm{I}^{\prime} \mathrm{H}_{2} \mathrm{O}_{2}$ ) ne fut pas significativement modifiée sous irradiation. Une corrélation a été observée entre l'augmentation de l'activité catalasique globale dans les cultures et la restauration d'une activité normale de la GAP-DH, probablement en rapport avec une diminution de l'oxydation des groupements SH.

ABSTRACT In vitro cell cultures were submitted to low doses of chronic external irradiation. Cultures were exposed for 8 days to ${ }^{60} \mathrm{Co}$ source by growing the cells in culture flasks, $10 \mathrm{~cm}$ above the irradiation source (dose rate : 6.25 mGy / day, i.e. 50 mGy /8 days). Human dermal fibroblast proliferation, protein and DNA contents were undisturbed under irradiation. In the same way, the transmembrane resting potential values of about 200 single cells, measured with a glass microelectrode, were similar in irradiated cells $(9.4$ $\pm 4.9 \mathrm{eV})$ and controls $(10.2 \pm 2.0 \mathrm{eV})$ on the 7 th day of the culture. Glucose metabolism was investigated through the activity of G6P-DH (a key enzyme of the pentose phosphate shunt) and the activities of GAP-DH and pyruvate kinase (key enzymes of glycolysis pathway). Assays performed over a complete growth curve showed that chronic irradiation did not induce significant change of G6P-DH but GAP-DH and pyruvate kinase appeared transiently inhibited (up to $25 \%$ ) during the early exponential growth phase. Catalase activity was not significantly perturbed under irradiation. A correlation between the increase of total catalase activity in cultures and the GAP-DH normal activity restoration was observed, probably related to a decrease of SH-group oxidation.

\footnotetext{
* Laboratoire de biologie cellulaire, Faculté de médecine PURPAN, 31073 Toulouse Cedex, France.

** Institut für Biochemie, Biophysik und Biologie, A-5020 Salzbourg, Autriche.
} 


\section{Introduction}

Si de très nombreux travaux ont été consacrés aux effets délétères des fortes doses de radiations ionisantes, délivrées de façon ponctuelle, très peu de recherches ont porté sur les effets biologiques des très faibles débits de doses d'irradiation délivrées de façon chronique soit tout au long d'une culture cellulaire soit au cours de la vie. Cependant, l'utilisation croissante de l'énergie nucléaire, des isotopes, le développement des vols à haute altitude et des vols spatiaux et la connaissance de notre environnement naturel ont accru l'intérêt porté à l'action de ces très faibles doses de rayonnements chroniques n'excédant pas quelques milligrays par an.

Ainsi, des études réalisées sur des végétaux cultivés sur terrain radioactif ont montré que des irradiations variant de 0,09 à $0,78 \mathrm{mrad} \cdot \mathrm{h}^{-1}$ $\left(0,9\right.$ à $\left.7,8 \mu \mathrm{Gy} \cdot \mathrm{h}^{-1}\right)$ pouvaient entraîner une stimulation du développement $[11,36]$. De même, Delone et al. [10] ont constaté une stimulation de la croissance de bulbes d'oignons qui avaient été soumis au rayonnement cosmique à bord de Cosmos 110 .

Au niveau des organismes unicellulaires, des expériences également réalisées à bord de satellites ont mis en évidence le rôle non négligeable du rayonnement cosmique. Kordium et al. [18] rapportent que la croissance de Proteus vulgaris, étudiée à bord de Soyouz 12, est plus rapide pendant les 48 premières heures de vol (dose absorbée d'environ 0,75 mGy). Par ailleurs, une stimulation de la prolifération de Paramecium aurelia, après 4 jours de culture à bord du laboratoire spatial soviétique Saliout 6, a été rapportée par Planel et al., Tixador et al., la dose journalière étant voisine de $0,11 \mathrm{mGy}[29,39]$.

Le problème posé par les effets de l'irradiation naturelle et des très faibles doses sur la prolifération cellulaire a été exploré de façon extensive par Planel et al. [29-30, 32-33, 37-39]. Un effet d'inhibition de la prolifération de paramécies a été démontré par des expériences effectuées sous radioprotection par des écrans de plomb [38] et dans le laboratoire souterrain de Moulis, Ariège [32], tandis qu'un effet de stimulation a été mis en évidence lors d'expériences d'irradiation [8], de recherches effectuées en altitude à Chamonix [37] ou dans le domaine spatial $[30,33,39]$.

Conter et al. [7] confirment ces résultats sur un autre modèle unicellulaire, Synechococcus lividus.

Les travaux effectués sur les mammifères ont mis en évidence une augmentation des aberrations chromosomiques dans des lymphocytes de lapins élevés sur un sol de haute radioactivité tellurique, $0,08 \mathrm{mGy} \cdot \mathrm{h}^{-1}$ [21] ou de populations humaines exposées à des niveaux de radioactivité naturelle (région de Guarapari, Brésil) de l'ordre de $0,03 \mathrm{mGy} \cdot \mathrm{h}^{-1}$ $[2,28]$; cependant, des études du même type sur les populations humaines vivant dans l'état du Kerala (Inde) n'ont abouti à aucun résultat significatif. 
Les travaux réalisés avec de faibles doses de rayonnement sur des cellules humaines en culture sont assez peu nombreux. Pour MacieiraCoelho et al., Azzarone et al. [25, 1], les rayonnements ionisants induisent un raccourcissement de la durée de vie des cellules cultivées in vitro qui pourrait être en rapport avec les radicaux libres générés par les rayonnements [17]. Cependant, Dobrachov et al., sur des fibroblastes normaux, et Diatloff et Macieira-Coelho, sur des fibroblastes humains provenant de donneurs normaux ou atteints de maladies génétiques, montrent que des doses variant de 1 à 5 Gy peuvent induire une stimulation de leur prolifération [13, 25].

L'extrapolation des résultats concernant les effets posés par les fortes doses est très contestable pour des faibles niveaux de dose et des faibles débits de dose comparables à ceux de l'irradiation naturelle. Le but de notre travail a été d'apporter des informations concernant les effets de la "dose maximale" à laquelle les travailleurs peuvent être éventuellement exposés "sans danger", la dose permissible étant actuellement de $50 \mathrm{mGy}$ par an. Croute et al. [9] ont démontré qu'une irradiation chronique ( 3 mois) à très faible débit de dose $(0,55$ mGy par jour, soit 50 mGy en 3 mois) entraîne une stimulation modérée de la prolifération cellulaire, qui devient significative après 16 repiquages, et qui s'accompagne d'une augmentation transitoire des activités enzymatiques liées à la destruction des produits de radiolyse (catalase, glutathion réductase).

Nous avons, pour notre part, comparé ces résultats aux effets d'une irradiation chronique de durée plus courte ( 8 jours), mais dont le débit de dose est 12 fois plus élevé (6,25 mGy/jour) entraînant la même dose absorbée en fin de culture que dans les expériences de Croute et al. [9] soit 50 mGy (dose annuelle permissible).

Les effets des rayonnements ionisants sont souvent en rapport avec une péroxydation des lipides membranaires [26] et/ou une oxydation des groupements SH liés aux membranes [27] pouvant entraîner une désorganisation structurale des lipoprotéines membranaires [42]. Par ailleurs, une augmentation de la consommation du glucose a été rapportée [15, 34]. Nous basant sur ces résultats, nous avons recherché les éventuelles modifications d'activité d'enzymes du métabolisme glucidique sous la dépendance de groupements SH (GAP-DH) ou en rapport plus ou moins étroit avec la réduction de produits oxydés sous irradiation (G6P-DH). Dans l'hypothèse d'une perturbation de la perméabilité membranaire liée à la péroxydation de ses lipides, la différence de potentiel transmembranaire a été mesurée. 


\section{Matériel et méthodes}

\section{Cultures stock}

La souche de fibroblastes humains utilisée ( $F$ 106) a été obtenue à partir d'explants de peau jeune provenant d'une chirurgie plastique mammaire. Les cellules ont été cultivées dans des flacons de $25 \mathrm{~cm}^{2}$ contenant $7,5 \mathrm{ml}$ de milieu de culture MEM (Eagle's minimum essential medium) supplémenté avec $5 \mathrm{~g} / \mathrm{l}$ de glucose, $20 \mu \mathrm{g} / \mathrm{ml}$ de gentamycine (Sigma) et $10 \%$ de sérum de veau fœtal (S.V.F. J.BIO). Les cultures ont été incubées à $37^{\circ} \mathrm{C} \pm 0,1^{\circ} \mathrm{C}$ dans une étuve à $\mathrm{CO}^{2}$.

\section{Etude de la prolifération cellulaire}

Un soin particulier a été pris dans le protocole expérimental pour que toutes les conditions de culture, excepté l'irradiation gamma, soient identiques dans les deux lots de cellules. Pour chaque expérience, $250 \mathrm{ml}$ de milieu de culture stérile contenant $10 \%$ de SVF ont été inoculés avec des fibroblastes provenant d'une culture cellulaire à confluence en vue d'obtenir une concentration finale de 30000 cellules $/ \mathrm{ml}$. Après agitation, le milieu a été réparti dans 32 flacons de $25 \mathrm{~cm}^{2}$, à raison de $7 \mathrm{ml}$ par flacon. Le premier lot (16 flacons) a servi de témoin, le deuxième lot (16 flacons) a été soumis à une irradiation gamma.

L'attachement des cellules a été contrôlé avant la mise sous irradiation et les numérations cellulaires ont été effectuées chaque jour, du $4^{e}$ au $8^{e}$ jour de culture, à l'aide d'un compteur Coultronics, modèle ZBI. Pour cela, les cellules ont été décollées de leur support plastique avec de la trypsine à $0,25 \%$ dans un tampon phosphate sans Ca ni Mg et contenant 0,02 \% EDTA. Des dilutions au $1 / 25^{e}$ ont été réalisées pour effectuer les numérations cellulaires. Pour chaque culture, 3 échantillons ont été prélevés et 4 comptages ont été réalisés par échantillon.

\section{Irradiation}

Les cultures cellulaires ont été exposées durant 8 jours à une source radioactive de ${ }^{60} \mathrm{CO}$ délivrant une dose de $6,25 \mathrm{mGy} / \mathrm{jour}$ au niveau des cultures, entraînant une dose globale de $50 \mathrm{mGy}$ au bout de 8 jours.

\section{Biochimie}

Du $4^{e}$ au $3^{e}$ jour de culture, après la numération cellulaire, les cellules ont été traitées en vue d'une analyse biochimique. Les cellules ont été centrifugées à $180 \mathrm{~g}$ durant $10 \mathrm{~min}$. Le culot cellulaire a été lavé avec du PBS puis recentrifugé ( 2 fois). Le culot final a été resuspendu dans $500 \mu \mathrm{l}$ de PBS et les cellules ont été cassées par sonication durant $30 \mathrm{~s}$. Les homogénats ont été centrifugés à $5000 \mathrm{~g}$ $\left(4^{\circ} \mathrm{C}, 5 \mathrm{~min}\right)$ et les surnageants, congelés à $-20^{\circ} \mathrm{C}$, ont été utilisés ultérieurement pour les dosages.

\subsection{Dosage des protéines}

La teneur en protéines a été déterminée selon la méthode de Lowry et al. [23], la courbe étalon étant établie à partir de sérum albumine bovine avec des concentrations comprises entre 0 et $100 \mathrm{mg} / \mathrm{ml}$. Le taux des protéines est exprimé en milligrammes de protéines par millilitre d'homogénat. 


\subsection{Dosage de l'ADN}

La teneur en $A D N$ a été obtenue selon la méthode fluorométrique de FiszerSzafarz [14] en utilisant l'acide 3-5 diaminobenzoique (DAB) et I'ADN de type 1 comme référence. Le taux d'ADN est exprimé en $\mu \mathrm{g} / 10^{6}$ cellules.

\subsection{Dosages enzymatiques}

L'activité catalasique a été appréciée en mesurant la vitesse de décomposition de $\mathrm{H}_{2} \mathrm{O}_{2}$ par spectrophotométrie U.V. à $240 \mathrm{~nm}$ pendant $5 \mathrm{~min}$ à $25^{\circ} \mathrm{C}$ [4]. L'activité catalasique est exprimée en unités internationales (UI) c'est-à-dire en micromoles de $\mathrm{H}_{2} \mathrm{O}_{2}$ dégradées par milligramme de protéines et par minute.

L'activité de la glucose-6-phosphate deshydrogénase (G6P-DH) a été dosée en mesurant, à $340 \mathrm{~nm}$, l'apparition du NADPH formé au cours de la réaction Lohr et Waller [22]. L'activité est exprimée en UI c'est-à dire en micromoles de NADPH formées par milligrammes de protéines et par minute.

L'activité de la glycéraldhéhyde-3-phosphate deshydrogénase (GAP-DH) a été évaluée en suivant à $340 \mathrm{~nm}$ l'oxydation du $\mathrm{NADH}$ selon la méthode de Udvardy et al. [40]. L'activité enzymatique est exprimée en micromoles de NAD détruites par milligramme de protéines et par minute (UI).

L'activité de la pyruvate kinase (PK) calculée à partir de la vitesse d'oxydation du NADH [16] est exprimée en micromoles de NADH détruites par milligramme de protéines et par minute.

\section{Mesure du potentiel de membrane}

Le potentiel transmembranaire a été mesuré [31] à l'aide de l'appareillage du $\operatorname{Pr}$ Steinhausler (Salzbourg, Autriche) schématiquement représenté dans la figure 1. La technique consiste à introduire dans la cellule, au moyen d'un micromanipulateur et sous observation microscopique, l'extrémité d'une microélectrode constituée par un capillaire de verre (diamètre $0,1 \mu \mathrm{m}$ ) empli d'une solution conductrice de $\mathrm{KCl} 3 \mathrm{M}$ lui conférant une résistance électrique de $5 \mathrm{Mohm}$. L'électrode intracellulaire et une électrode de référence (plongée dans le milieu extracellulaire) sont reliées à un amplificateur et les données sont visualisées sur un oscilloscope et enregistrées sur un enregistreur graphique. On considère comme zéro la faible différence de potentiel enregistrée lorsque la microélectrode plonge dans le même milieu que l'électrode de référence. Ce n'est qu'au moment où la pointe de la microélectrode traverse la membrane cellulaire qu'apparaît brusquement une différence de potentiel entre les électrodes. Environ 100 cellules ont été testées pour chaque condition de culture.

\section{Analyses statistiques}

Les résultats correspondent aux valeurs moyennes d'au moins trois échantillons plus ou moins l'erreur standard. 


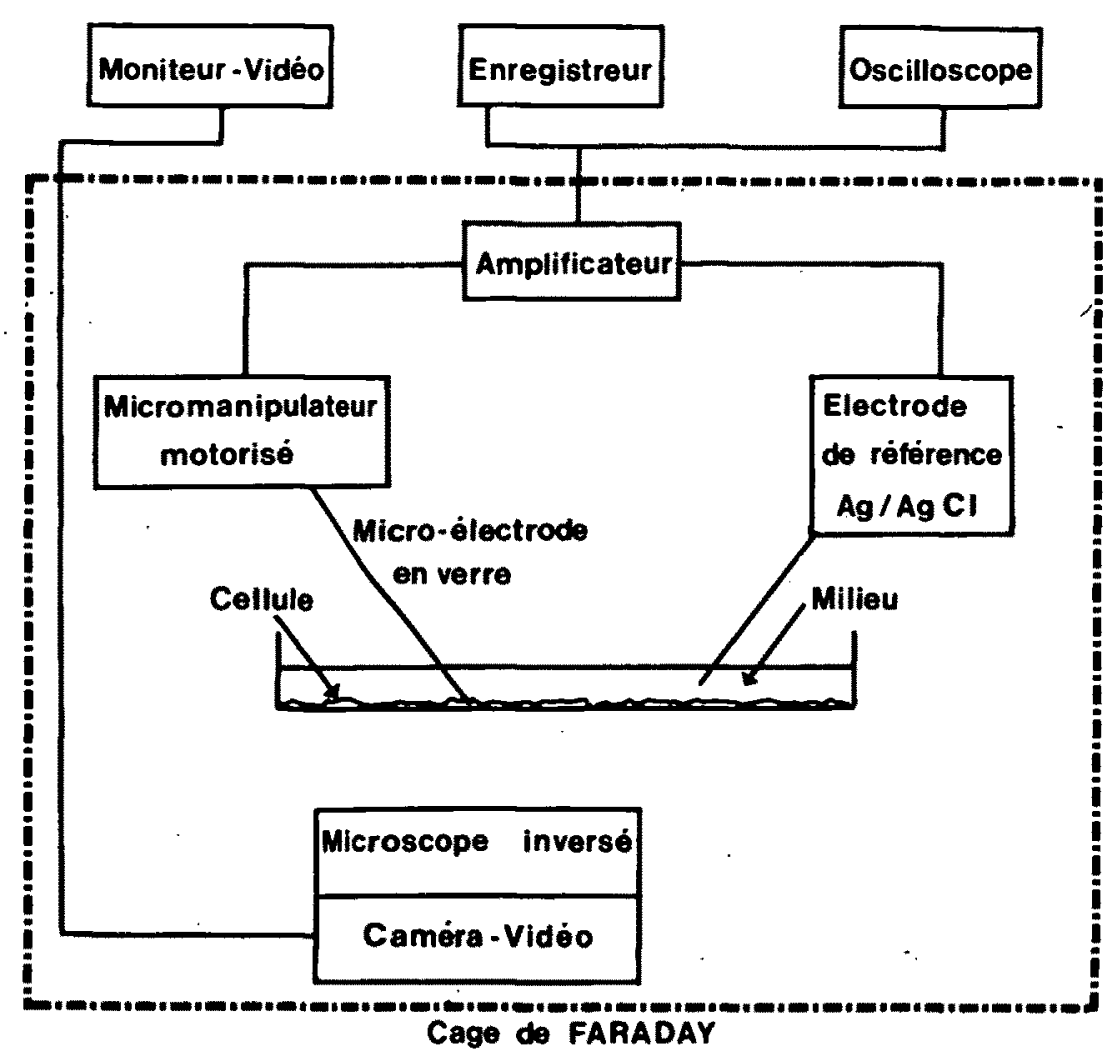

Fig. 1. - Dispositif expérimental utilisé pour la mesure du potentiel de membrane de fibroblastes dermiques humains (Laboratoire de biochimie, biophysique et biologie, Pr Steinhausler, Salzbourg, Autriche).

Experimental device for the measurement of transmembrane potential of human skin fibroblasts.

\section{Résultats}

\section{Etude de la prolifération cellulaire}

La figure 2 montre l'effet d'une irradiation chronique gamma $\left({ }^{60} \mathrm{Co}\right)$ à un débit de dose de $6,25 \mathrm{mGy} / \mathrm{jour}$ sur la prolifération in vitro de fibroblastes dermiques humains normaux et sur la teneur en ADN des cellules après 8 jours d'irradiation. La prolifération cellulaire a été contrôlée quotidiennement du $4^{\mathrm{e}}$ au $8^{\mathrm{e}}$ jour, c'est-à-dire pour des cellules ayant reçu des doses respectives de 27 - 31,25 - 37,50 - 43,75 et' 50 mGy. Certaines cultures ont été renourries au $5^{\mathrm{e}}$ jour afin de prolonger la durée de la phase exponentielle.

Comme le montre figure 2, la croissance des cultures non renourries (courbe $A$ ) est tout à fait comparable dans les témoins et sous irradiation avec une phase exponentielle rapide (les taux de croissance respectifs des témoins et des irradiées étant de 2,01 $\pm 0,09$ et 1,96 $\pm 0,13$ ) suivie au $5^{e}$ jour par une phase de décélération. Le changement de milieu au 
$5^{\mathrm{e}}$ jour (courbe $B$ ) est suivi par une reprise rapide du taux de croissance aussi bien dans les cultures témoins $(1,70 \pm 0,05)$ que dans les cultures irradiées $(1,65 \pm 0,11)$. Les variations du nombre de cellules entre cultures témoins et irradiées ne dépassent pas $6 \%$ tout au long de la culture et l'étude statistique ne met en évidence aucune différence significative. On peut, cependant, observer une plus grande variabilité dans les cultures irradiées.

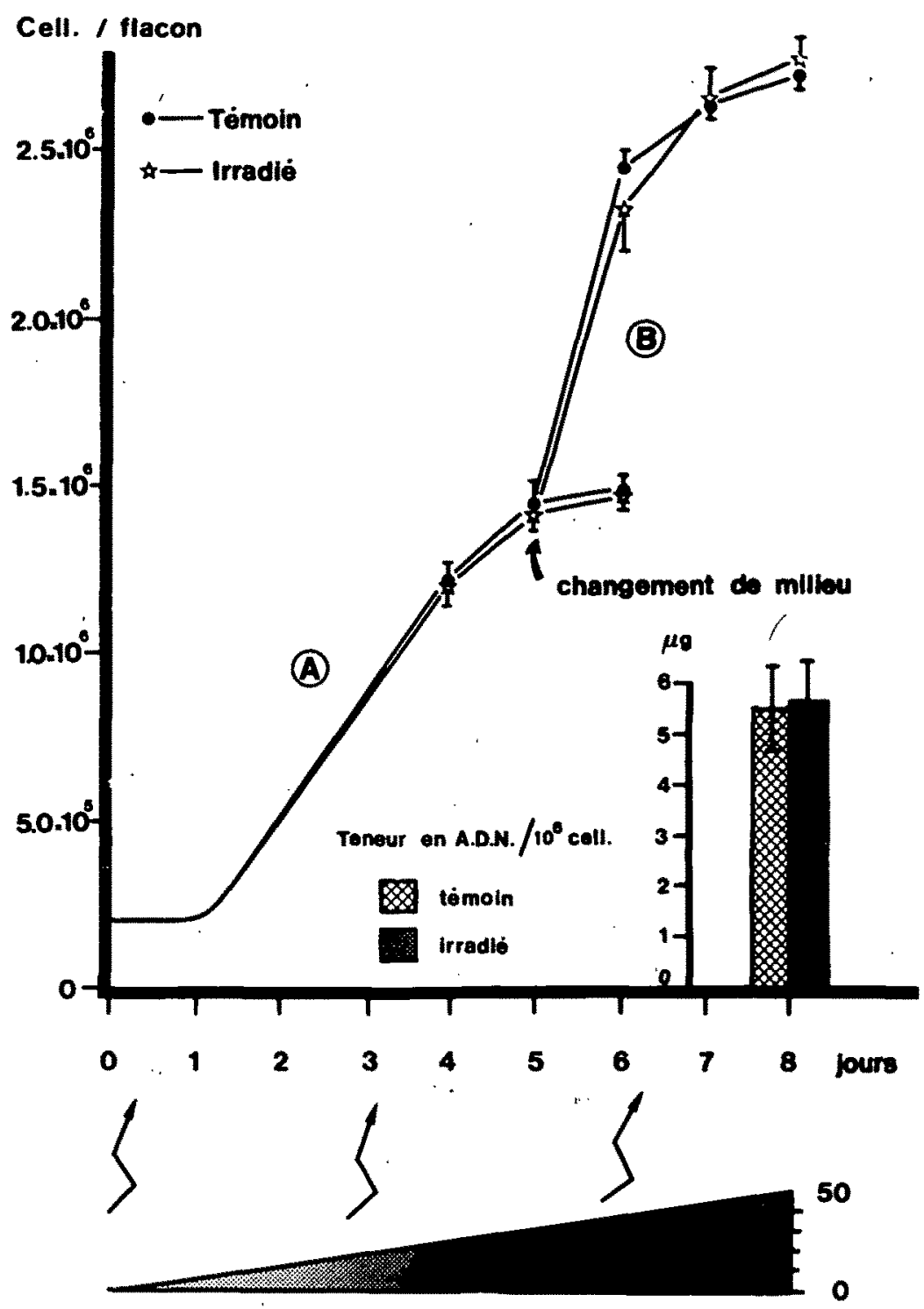

Dose absorbie (mGy)

Fig. 2. - Influence d'une irradiation externe chronique $\left({ }^{60} \mathrm{Co}\right)$ à faible débit de dose (6,25 mGy/jour) sur la prolifération et la teneur en ADN de fibroblastes humains dermiques.

Effect of chronic external irradiation $\left({ }^{60} \mathrm{Co}\right)$ at low dose rate $(6.25 \mathrm{mG} / \mathrm{d})$ on the proliferation and DNA content of human skin fibroblasts. 
La quantité moyenne d'ADN (calculée pour $10^{6}$ cellules) au $8^{e}$ jour de culture (début de phase plateau) ne présente pas de différence significative entre les fibroblastes témoins et irradiés (fig. 2). Les teneurs en protéines totales ( $\mu \mathrm{g} / 10^{6}$ cellules) (tab. I) apparaissent assez stables tout au long des cultures. Leurs valeurs varient de $225 \pm 7$ à $242 \pm 10$ pour les cellules témoins et de $210 \pm 22$ à $251 \pm 8$ pour les cellules irradiées.

\section{Analyses biochimiques (tab. II)}

L'activité de la catalase (enzyme de défense contre les péroxydes) augmente régulièrement pendant la phase de croissance exponentielle du $4^{e}$ au $6^{e}$ jour pour diminuer du $7^{e}$ au $8^{e}$ jour (phase plateau). Ces variations en fonction du temps sont similaires dans les cultures témoins et irradiées et aucune modification significative n'a été mise en évidence quelles que soient les expériences.

D'autre part, nous avons étudié trois enzymes du métabolisme du glucose : la G6P-DH, enzyme clé de la voie oxydative des pentoses, la GAP-DH et la PK, enzymes clés de la glycolyse. L'activité de la G6P-DH des cultures témoins, étudiée quotidiennement, augmente progressivement de $52,20+0,50$ UI ( $4^{e}$ jour) à $80,52+3,46$ UI le $7^{e}$ jour. L'activité décroît le dernier jour de culture. Pour les cellules irradiées, la G6P-DH varie de façon parallèle et ne montre aucune différence significative avec les cultures témoins.

\section{TABLEAU I}

Effet d'une irradiation chronique externe $\left({ }^{60} \mathrm{Co}\right)$ à taible débit de dose $(6,25 \mathrm{mGy} / \mathrm{jour})$ sur la prolifération et la teneur en protéines totales de fibroblastes dermiques humains en culture. Les valeurs des protéines sont données en $\mu \mathrm{g}$ pour $10^{6}$ cellules.

Effect of chronic external irradiation $\left({ }^{60} \mathrm{Co}\right)$ at low dose rate $(6.25 \mathrm{mG} / \mathrm{d})$

on the proliferation and total protein contents of human skin fibroblasts in vitro (protein values in $\mu \mathrm{g} / 10^{6}$ cells)

\begin{tabular}{|c|c|c|c|c|c|c|}
\hline \multicolumn{2}{|c|}{ JOURS DE CULTURE } & qème & 5ème & Gème & 7ème & Bème \\
\hline $\begin{array}{l}N \\
U \\
M \\
E \\
R \\
A\end{array}$ & $\begin{array}{l}\text { TEMOINS } \\
\text { IRRADIES }\end{array}$ & $\begin{array}{l}1214044 \pm 45248 \\
1186333 \pm 66289\end{array}$ & $\begin{array}{l}1424027 \pm 73657 \\
1399676 \pm 53544\end{array}$ & $\begin{array}{l}1359895 \pm 61351 \\
1472187 \pm 58248\end{array}$ & & \\
\hline $\begin{array}{l}T \\
1 \\
0 \\
N \\
S\end{array}$ & $\begin{array}{l}\text { TEMOINS } \\
\text { IRPADIES }\end{array}$ & $\begin{array}{l}\text { Cultures renourri } \\
\text { au 5ème jour }\end{array}$ & es & $\begin{array}{l}2433125 \pm 56000 \\
2310000 \pm 128000\end{array}$ & $\begin{array}{l}2625178 \pm 36340 \\
2641899 \pm 91880\end{array}$ & $\begin{array}{l}2697639 \pm 45285 \\
2748472 \pm 73080\end{array}$ \\
\hline $\begin{array}{l}\text { PRO- } \\
\text { TEF } \\
\text { NES }\end{array}$ & $\begin{array}{l}\text { TEMOINS } \\
\text { IRRADIES }\end{array}$ & $\begin{array}{l}227 \pm 19 \\
210 \pm 22\end{array}$ & $\begin{array}{l}228 \pm 7 \\
238 \pm 12\end{array}$ & $\begin{array}{l}225 \pm 7 \\
224 \pm 11\end{array}$ & $\begin{array}{l}242 \pm 10 \\
251 \pm 8\end{array}$ & $\begin{array}{l}237 \pm 13 \\
232 \pm 10\end{array}$ \\
\hline
\end{tabular}


TABLEAU \|

Activités enzymatiques (par mig de protéines) de fibroblastes dermiques humains cultivés sous irradiation chronique $\left({ }^{60} \mathrm{Co}\right)$ à faible débit de dose $(6,25 \mathrm{mGy} / \mathrm{jour})$. Enzymatic activities of human skin fibroblasts in vitro under chronic irradiation $\left({ }^{60} \mathrm{Co}\right)$ at low dose rate $(6.25 \mathrm{mGy} / \mathrm{d})$.

\begin{tabular}{|c|c|c|c|c|c|c|}
\hline $\begin{array}{c}\text { ACTMTIES } \\
\text { ENZYMATIQUES } \\
\text { (U.I.) }\end{array}$ & $\begin{array}{l}\text { JOURS DE } \\
\text { CULTURE }\end{array}$ & . & 5ème & Gème & 7ème & 8ème \\
\hline CATALASE & $\begin{array}{l}\text { TEMOINS } \\
\text { IRRADIES }\end{array}$ & $\begin{array}{r}9.43 \pm 0.96 \\
10.29 \pm 1.65\end{array}$ & $\begin{array}{l}13.74 \pm 0.62 \\
12.88 \pm 0.58\end{array}$ & $\begin{array}{l}13.21 \pm 0.87 \\
13.29 \pm 0.67\end{array}$ & $\begin{array}{l}10.75 \pm 0.22 \\
10.77 \pm 0.45\end{array}$ & $\begin{array}{l}9.88 \pm 0.38 \\
9.82 \pm 0.51\end{array}$ \\
\hline $\begin{array}{c}\text { ACTNITE } \\
\text { GLOBALE } \\
\text { CATALASIOUE/ } \\
\text { CULTURE } \\
\end{array}$ & $\begin{array}{l}\text { TEMOINS } \\
\text { IRRADIES }\end{array}$ & $\begin{array}{l}2.569 \\
2.550\end{array}$ & $\begin{array}{l}4.448 \\
4.232\end{array}$ & $\begin{array}{l}7.220 \\
6.877\end{array}$ & $\begin{array}{l}6.620 \\
7.139\end{array}$ & $\begin{array}{l}6.317 \\
6.261\end{array}$ \\
\hline GEP-DH & $\begin{array}{l}\text { TEMOINS } \\
\text { IRRADIES }\end{array}$ & $\begin{array}{l}52,20 \pm 0.50 \\
53,20 \pm 3.10\end{array}$ & $\begin{array}{l}74.36 \pm 1,93 \\
67.77 \pm 3.88\end{array}$ & $\begin{array}{l}79.12 \pm 2.05 \\
78.87 \pm 4.30\end{array}$ & $\begin{array}{l}80.52 \pm 3.46 \\
78.78 \pm 7.65\end{array}$ & $\begin{array}{l}73.61 \pm 4.70 \\
69.01 \pm 1.00\end{array}$ \\
\hline GAP-DH & $\begin{array}{l}\text { TEMOINS } \\
\text { IRPADIES }\end{array}$ & $\begin{array}{c}219.00 \div 18.20 \\
178.10 \pm 21.10^{*}\end{array}$ & $\begin{array}{l}234,30 \pm 22,70 \\
229,60 \pm 15,30\end{array}$ & $\begin{array}{l}412.52 \pm 23.43 \\
415.20 \pm 37.70\end{array}$ & $\begin{array}{l}480,00 \pm 45,20 \\
460,00 \pm 42.10\end{array}$ & $\begin{array}{l}470.05 \pm 39.51 \\
475.50 \pm 42.73\end{array}$ \\
\hline $\begin{array}{l}\text { PYRUVATE } \\
\text { KINASE }\end{array}$ & $\begin{array}{l}\text { TEMOINS } \\
\text { IRRADIES }\end{array}$ & $\begin{array}{c}355.08 \pm 24.42 \\
272.84 \pm 35.55^{\circ}\end{array}$ & $\begin{array}{c}564.30 \pm 41.74 \\
426.80 \pm 75.80\end{array}$ & $\begin{array}{l}634.77 \pm 36.72 \\
574.00 \pm 51.88\end{array}$ & $\begin{array}{l}810.50 \pm 39.90 \\
778.70 \pm 107.0\end{array}$ & $\begin{array}{l}607.38 \pm 62.95 \\
580.23 \pm 33.74\end{array}$ \\
\hline
\end{tabular}

Le tableau II montre que l'activité de la GAP-DH varie de façon importante au cours de la culture. Dans les cultures non renourries ( $4^{\mathrm{e}}-5^{\mathrm{e}}$ jour), l'activité est faible et apparaît inhibée sous irradiation et de façon significative au $4^{e}$ jour de culture. Dans les cultures renourries au $5^{e}$ jour, l'activité est environ 2 fois plus élevée dans les deux conditions de culture, mais il n'apparaît aucune différence significative entre témoins et irradiées.

L'activité de la $\mathrm{PK}$ pour les cellules témoins augmente régulièrement de $355,08 \pm 24,42$ UI à $810,50 \pm 39,90$ Ul jusqu'au $7^{e}$ jour. On observe une inhibition constante de son activité sous irradiation de l'ordre de 4 à $25 \%$, inhibition qui n'est cependant significative que le $4^{e}$ et $5^{e}$ jour.

\section{Etude du potentiel de membrane}

Deux cents cellules ont été individuellement testées (100 cellules témoins et 100 cellules irradiées). La valeur du potentiel de membrane des cellules témoins est sensiblement constante tout au long de la culture $\left(10,5 \pm 1,8 \mathrm{eV}\right.$ le $4^{\mathrm{e}}$ jour, $10,2 \pm 2,0 \mathrm{eV}$ le $7^{\mathrm{e}}$ jour $)$. Des valeurs comparables ont été obtenues pour les cellules irradiées $(9,9 \pm 2,3 \mathrm{eV}$ le $4^{e}$ jour et $9,4 \pm 4,9 \mathrm{eV}$ le $7^{e}$ jour). Les différences entre cultures témoins et irradiées ne sont pas significatives, mais on observe une plus grande 
hétérogénéité des valeurs sous irradiation comme le montrent les valeurs des erreurs standards.

\section{Discussion, conclusion}

Nos résultats montrent qu'une irradiation externe chronique de 8 jours $\left({ }^{60} \mathrm{Co}\right)$, délivrant une dose totale de $50 \mathrm{mGy}(6,25 \mathrm{mGy} / \mathrm{jour})$, n'entraîne aucune modification significative de la prolifération, du taux des protéines totales et de la quantité d'ADN de fibroblastes humains dermiques en culture in vitro. Nous n'avons pas retrouvé les effets de stimulation obtenus par Croute et al. [9] sur des fibroblastes humains de poumon irradiés pendant 3 mois avec une source de thorium (débit de dose 0,50 mGy/jour). Cette divergence de résultats peut être en rapport avec une différence de sensibilité des souches cellulaires utilisées, mais aussi avec le débit de dose et/ou la source d'irradiation.

En effet, Budinger et Luckey $[6,24]$ ont suggéré que le débit de dose était probablement plus important que la dose totale délivrée lors d'une irradiation. Cette affirmation se vérifie si on regarde la littérature concernant des irradiations faites sur différentes espèces animales et notamment la souris et le rat [19-20]. D'autre part, Sacchetti et al. [35] ont montré que les cellules de cochon d'Inde irradiées par 2 doses de 25 mGy à 4 jours d'intervalle donnent des résultats différents de ceux obtenus pour des cellules irradiées avec une seule dose de $50 \mathrm{mGy}$. Par ailleurs, Verma [41] montre que, vis-à-vis de lipides polyinsaturés de liposomes et pour une même dose d'irradiation de $30 \mathrm{mGy}$, les rayons $X$ ont une efficacité biologique relative (EBR) plus importante que ceux du ${ }^{60} \mathrm{Co}$. L'absence de stimulation de la prolifération dans nos expériences d'irradiations chroniques par le ${ }^{60} \mathrm{Co}$ pourrait être en faveur d'une EBR plus faible du ${ }^{60} \mathrm{Co}$ par rapport à celle du thorium $\left(\alpha, \beta^{-}, \gamma\right)$.

Un certain consensus s'est établi sur le fait que les rayonnements ionisants entraînent une diminution des groupements $\mathrm{SH}$ membranaires qui pourrait être responsable de l'inactivation d'enzymes liées à la membrane et perturber le transport transmembranaire de substances variées. Plus particulièrement, Palecz et Leyko [27] mettent en évidence une diminution de l'activité ATPasique membranaire $\mathrm{Na}^{+} / \mathrm{K}^{+}$dépendante après une irradiation de $50 \mathrm{mGy}$, ce qui pourrait laisser supposer une perturbation du transport actif du $\mathrm{Na}^{+}$vers l'extérieur et du $\mathrm{K}^{+}$vers l'intérieur de la cellule et, ainsi, modifier le potentiel transmembranaire. Pour les faibles débits de dose utilisés dans nos expériences, 6,25 mGy/jour, nous n'avons observé aucune modification significative du potentiel de membrane de fibroblastes irradiés pendant 7 jours.

Dans nos conditions expérimentales, l'activité de la voie de la glycolyse (mise en évidence par les activités de la GAP-DH et de la PK) est transitoirement inhibée pendant la phase exponentielle de croissance ( $4^{e}$ jour de culture). La diminution d'activité de la GAP-DH pourrait s'expliquer par l'oxydation du groupement $\mathrm{SH}$ qui se trouve sur le site actif de l'enzyme. De même Bartoc et al. [3] ont trouvé une diminution d'activité des 
enzymes de la glycolyse (hexokinase et phosphofructokinase) qui est liée à l'oxydation des groupements $\mathrm{SH}$. La diminution d'activité de la pyruvate kinase (enzyme irréversible de la voie de la glycolyse) pourrait être le résultat de la diminution de son substrat due à la diminution d'activité des enzymes situées en amont dans la voie de la glycolyse. On peut penser que l'effet d'inhibition de la GAP-DH, qui est transitoire, est due aux produits de radiolyse : $\mathrm{OH}^{\circ}, \mathrm{O}_{2}^{\bar{\beta}}$ et $\mathrm{H}_{2} \mathrm{O}_{2}$ générés dans la cellule et dans le milieu de culture. Ces produits ne seraient pas complètement détruits, en début de culture, en raison de la faible concentration cellulaire et, par conséquence, en raison d'une activité catalasique (et péroxydasique) globale insuffisante (environ 2,56 UI au $4^{e}$ jour). Ces radicaux libres ou $\mathrm{I}^{\prime} \mathrm{H}_{2} \mathrm{O}_{2}$, en oxydant le groupement SH présent sur le site actif de la GAP$\mathrm{DH}$, empêchent l'activité normale de l'enzyme. Au cours de la culture, l'activité enzymatique globale de la culture varie en fonction du nombre de cellules et en fonction de leur activité propre (tab. II). L'activité globale catalasique atteint son maximum au $6^{e}$ jour de culture et elle est 2,8 fois plus importante qu'au début de la culture. On peut supposer que cette activité est alors suffisante pour entraîner la destruction des radicaux libres et des péroxydes générés par ces faibles débits de dose et permettre ainsi une activité normale de la GAP-DH. La restauration de l'activité de la GAP-DH entraîne la production d'un taux normal de substrat pour la pyruvate kinase.

L'activité de la voie des pentoses phosphates, dont la G6P-DH est une enzyme clé, n'apparaît pas modifiée, contrairement à nos hypothèses. En effet, plusieurs auteurs (Roberts et al., Croute et al., Biaglow et al.) ont montré que la réduction des péroxydes par la glutathion péroxydase, au dépend d'une oxydation du NADPH, peut se traduire par une stimulation de la voie des pentoses phosphates $[5,9,34]$.

\section{RÉFÉRENCES}

[1] AzZaRone B., diatloff-Zito C., BILlaRdon C., MACIEIRA-COElHo A. Effect of low dose rate irradiation on the division potential of cells in vitro. VII. Human fibroblasts from young and adults donnors. In vitro, 1979, 16, 634-638.

[2] BARCINSKI M.A., BREU M.C., ALMEIDA J.C., NAYA J.M., FONSECA L.C.G., CASTRO L.E. - Cytogenetic investigations in a brasilian population living in an area of high natural radiation. Am J. Hum. Genet., 1975, 27, 802-805.

[3] BARTOC R., BRUHIS S., KLEIN R., MOLDOVEANU E., OERIU I., OERIU S. Effect of age and $\mathrm{SH}$ active groups on the activity of some enzymes involved in the carbohydrate metabolism. Exp. Geront., 1975, 10, 161-164.

[4] BEERS R.F., SIZERS I.W. - A spectrophotometric method for measuring the breakdown of hydrogen peroxide by catalase. J. Biol. Chem., 1952, 195, 133-140.

[5] BIAGLOW J.E., TUTTLE S.W., CERNIGLIA G.J., HELD K., EPP Ed. - The importance of hydrogen peroxide in the aerobic radiation response ; the role of glutathione peroxidase, catalase and the pentose cycle. In : Congress abstracts of Radiation research, Toronto, 7-12 July 1991 (Chapman J.D., Dewey W.C., Whitmore G.F., Eds.), New York : Academic Press, 1991, Vol. 1, p. 208. 
[6] BUDINGER T.F. - Frontal attack on radiation. Science, 1970, 168, p. 315.

[7] CONTER A., DUPOUY D., PLANEL H. - Demonstration of a biological effect of natural ionizing radiations. Int. J. Radiat. Biol., 1983, 43, 421-432.

[8] CROUTE F., SOleilhavouP J.P., VIDAL S., DUPOUY D., PLANEL H. Paramecium tetraurelia growth stimulation under low-level chronic irradiation, investigations on a possible mechanism. Radiat. Res., 1982, 92, 560-567.

[9] CROUTE F., VIDAL S., SOLEILHAVOUP J.P., VINCENT C., SERRE .G., PLANEL $\mathrm{H}$. - Effects of a very low dose rate of chronic ionizing radiation on the division potential of human embryonic lung fibroblasts in vitro. Exp. Geront., 1986, 21, 1-11.

[10] DELONE N.L., MOROZOVA E.M., ANTINOV V.V., PARFENOY G.P., TRUSOVA A.S. - Stimulation of the growth in the onion allium cepa after storage of the bulb during space flight on the Sputnik Cosmos 110. Nucl. Sci. Abst. 1968, 22, p. 23676 .

[11] DELPOUX M. - Etude expérimentale des effets de la radioactivité naturelle tellurique sur les végétaux. Hypothèse sur l'influence de l'environnement fortement énergétique sur les êtres vivants. Thèse Doctorat d'Etat, Toulouse, 1974, $n^{\circ} 631$.

[12] DIATLOFF C. MACIEIRA-COELHO A. - Effect of low dose rate irradiation on the division potential of cells in vitro. $V$. Human skin fibroblasts from donors with a high risk of cancer. J. Natl. Cancer Inst., 1979, 63, 55-59.

[13] DOBRACHOV YU. P., ANDREYEV S.V. - Action des faibles doses d'irradiation gamma sur la croissance de fibroblastes normaux. Radiobiologiya. 1974, 14, 443446.

[14] FISZER-SZAFARZ B., SZAFARZ D., GUEVARA de MURILLO A. - A general, fast, and sensitive micromethod for DNA determination : application to rat and mouse liver, rat hepatoma, human leukocytes, chicken fibroblasts, and yeast cells. Anal. Biochem., 1981, 110, 165-170.

[15] FOMENKO B.F., AKOEV I.G. - Effect of ionizing radiation on transmembrane potential of Streptococcus. Radiat. Res. 1979, 77, 479-489.

[16] GUTMAN I., BERNT E. - Pyruvate kinase assay in serum and erythrocytes. In : "Methods of enzymatic analysis" (Bergmeyer, H.U., Ed.) New York : Academic press, 1974, Vol. 2, 774-778.

[17] HARMAN D. - The aging process. Proc. Natl. Acad. Sci. 1981, 78, 7124-7128.

[18] KORDIUM V.A., POLIDOVA L.V., MASHINSKY A.L., MANKO V.G., NECHITAILO G.N., SUSANOV D.V., KONSHIN N.I. - Peculiarities of microorganism's growth in extreme conditions. COSPAR 17th plenary meeting, Sao Paulo, June-July 1974, 254-255.

[19] KOZNOVA L.B., ZAYTSEVA R.N., TIKHOMIROVA L.K. - Changes in total quantity of marrow cells and their mitotic index in rats following brief and prolonged gamma irradiation. Nucl. Sci. Abstr. 1972, 30, p. 9951.

[20] LANGHAM W.H. - Implications of space radiations in manned space flights. Aerosp. Med., 1959, 30, 410-412.

[21] LEONARD A., DELPOUX M., DECAT G., LEONARD E.D. - Natural radioactivity in southwest France and its possible genetic consequences for mammals. Radiat. Res. 1979, 77, 170-181.

[22] LOHR G.W., WALLER H.D. - Glucose-6-phosphate dehydrogenase. In : "Methods of enzymatic analysis" (Bergmeyer H.U., Ed.) New York : Academic Press, 1974, 643-646.

[23] LOWRY O.H., ROSEBROUGH N.J., RANDALL R.J. - Protein measurement with the Folin phenol reagent. J. Biol. Chem., 1951, 193, 265-275.

[24] LUCKEY T.D. - Hormesis with ionizing radiation. Boca Raton : CRC Press, 1980, p. 42. 
[25] MACIEIRA-COELHO A., DIATLOFF C., BILLARDON C., BOURGEOIS C.A., MALAISE E. - Effect of low dose rate ionizing radiation on the division potential of cells in vitro. III. Human lung fibroblasts. Expl. Cell. Res., 1977, 104, 215-221.

[26] MYERS D.K., SLADE D.E. - Radiosensitization of mammalian cells by iodoacetamide and related compounds. Radiat. Res., 1967, 30, 186-203.

[27] PALECZ D., LEYKO W. - Effect of gamma radiation on enzymatic and sulphydryl groups of human erythrocyte membrane. Int. J. Radiat. Biol., 1983, 44, 293-299.

[28] PENNA FRANCA E. - Review in Brasilian investigations in areas of high natural radioactivity. II. Internal exposure and cytogenetic survey. In : International symposium on areas of high natural radioactivity, Pocos de Caldas, Brasil, 16-20 June 1975 (CULLEN T.L., PENNA FRANCA E., Eds.). Rio de Janeiro : Academia Brasileria de Ciençias, 1977, 29-48.

[29] PLANEL H., TIXADOR R., NEFEdOV I.G., GRETCHKO G., RICHOILley G. Preliminary results of Cytos experiment flown in Salyut 6 : investigations on Paramecium aurelia. Life Sci. Space Res., 1979, 17, 139-144.

[30] PLANEL H., TIXADOR R., NEFEDOV Y., GRETCHKO G., RICHOILLEY G. Effects of space flight factors at the cellular level : results of the Cytos experiment. Aviat. Space Environ. Med., 1982, 53, 370-374.

[31] REDMANN K. - Messung elektrischer Membranpotentiale kultivierter Einzelzellen mittels Mikroelektroden. Acta Biol. Med. Germ., 1971, 27, 55-69.

[32] RICHOILLEY G., PLANEL H., TIXADOR R. - Action biologique des radiations ionisantes naturelles - étude de la cinétique de la Paramécie cultivée en laboratoire souterrain. Ann. Spéléo, 1973, 28, 391-403.

[33] RICHOILLEY G., TEMPLIER J., BES J.C., GASSET G., PLANEL H., TIXADOR R. - Effets biologiques de l'impesanteur à l'échelon cellulaire. Etude comparative de cultures de paramécies à bord de la station orbitale Saliout 6 et d'un ballon stratosphérique. C.R. Acad. Sci. Paris, 1984, 20, 845-848.

[34] ROBERTS W., KARTHA M., SAGONE L.S. - Effect of irradiation on the hexose monophosphate shunt pathway of human lymphocytes. Radiat. Res. 1979, 79, 601-610.

[35] SACCHETTI C., DIENA F., FIESCHI A. - Bone-marrow impairment and recovery following total body X-irradiation. In : "Immediate and low level effects of ionizing radiations" (Buzzati-Traverso, A.A., Ed.). London : Taylor and Francis, 1960, 139145.

[36] SAROZIEK J. - Radioekologia Marchantia polymorpha L.W. Sudetach. Monogr. Bot. Pol., 1972, 35, 161-203.

[37] SOLEILHAVOUP J.P., CROUTE F., TIXADOR R., BLANQUET Y., PLANEL H. Influence du rayonnement cosmique à très haute altitude sur la durée du cycle cellulaire. C.R. Soc. Biol., 1975, 169, 426-429.

[38] TIXADOR R., PLANEL H., RICHOILLEY G., TOLLON - Etude de la durée du cycle cellulaire chez Paramecium aurelia cultivée en environnement normal ou sous radioprotection. C.R. Soc. Biol., 1969, 163, 1429-1432.

[39] TIXAdOR R., RICHOILley G., GRETCHKO G., NEFEDOV Y., PLANEL H. Multiplication de Paramecium aurelia à bord du vaisseau spatial Saliout 6 (expérience Cytos). C.R. Acad. Sc., 1978, 287, 829-832.

[40] UDVARDY J., BALOGH A., FARKAS G.L. - Modulation of glyceraldehyde-3-phosphate dehydrogenase in Anacytis nidulans by glutathione. Arch. Microbiol., 1982, $133,2-5$.

[41] VERMA S.P. - Low levels of irradiation modify lipid domains in model membranes : a laser Raman study. Radiat. Res. 1986, 107, 183-193.

[42] YONEI S., KATO M. - X-ray-induced structural changes in erythrocyte membranes studied by the use of fluorescent probes. Radiat. Res., 1978, 75, 31-45. 